\title{
Synchronous Bilateral Breast Carcinoma
}

National Cancer Institute

\section{Source}

National Cancer Institute. Synchronous Bilateral Breast Carcinoma. NCI Thesaurus. Code C40370.

Carcinoma that is detected in one breast within two months from the diagnosis of carcinoma in the other breast. 